\title{
Verbal and Nonverbal Emotional Memory Following Unilateral Amygdala Damage
}

\author{
Tony W. Buchanan, ${ }^{1}$ Natalie L. Denburg, Daniel Tranel, and Ralph Adolphs \\ Department of Neurology, Division of Cognitive Neuroscience, University of Iowa, College of Medicine, Iowa City, Iowa 52242, USA
}

\begin{abstract}
The amygdala is involved in the normal facilitation of memory by emotion, but the separate contributions of the left and right amygdala to memory for verbal or nonverbal emotional material have not been investigated. Fourteen patients with damage to the medial temporal lobe including the amygdala (seven left, seven right), 18 brain-damaged, and 36 normal controls were exposed to emotional and neutral pictures accompanied by verbal narratives. Memory for both narratives and pictures was assessed with a free recall test $24 \mathrm{~h}$ later. Subjects with left amygdala damage failed to show the normally robust enhancement of memory for verbal and nonverbal emotional stimuli. The group with right amygdala damage showed the normal pattern of facilitation of memory by emotion for both verbal and nonverbal stimuli despite an overall reduction in memory performance. Furthermore, subjects with left amygdala damage were disproportionately impaired on memory for emotional narratives as compared with memory for emotional pictures. The latter finding offers partial support for a lateralized and material-specific pattern of the amygdala's contribution to emotional memory.
\end{abstract}

By virtue of its location and connectivity in the brain, the amygdala has been the focus of much interest as a possible component of the neural system subserving learning and memory (Squire 1987; Zola-Morgan et al. 1991). It was originally thought to have an integral role in the formation of declarative memories. More careful study has revealed the role of the amygdala to be separate from the traditional "medial temporal lobe memory system" that comprises the hippocampus and adjacent cortex (Zola-Morgan et al. 1986, 1991; Murray 1992). Instead, there is now evidence that the amygdala has a modulatory rather than an essential role in declarative memory formation, a role that depends on whether or not the memory involves emotional arousal. Based on the work of McGaugh and colleagues, it is proposed that a neurobiological system arises through an interaction between stress hormones (both catecholamines and glucocorticoids) released during emotional events and the amygdaloid complex that results in increased memory for these events (McGaugh et al. 1990; McGaugh and Cahill 1997; McGaugh 2000).

In normal populations, emotionally arousing stimuli are consistently remembered better than neutral stimuli (Heuer and Reisberg 1990; Bradley et al. 1992; Buchanan and Lovallo 2001). Individuals with amygdala damage, however, do not show this effect (Adolphs et al. 1997, 2000; Phelps et al. 1997; LaBar and Phelps 1998), an impairment that is particularly clear in rare case studies of patients with selective bilateral amygdala damage (Cahill et al. 1995; Adolphs

${ }^{1}$ Corresponding author.

E-MAIL tony-buchanan@uiowa.edu; FAX (319) 353-8737.

Article and publication are at http://www.learnmem.org/cgi/doi/ $10.1101 / \mathrm{lm} .40101$. et al. 1997). Such lesion studies, along with functional neuroimaging research in normal individuals (Cahill et al. 1996; Hamann et al. 1999; Canli et al. 2000), have illustrated that the amygdala is involved in the encoding and/or consolidation of long-term declarative memories for emotional stimuli. For example, in a recent fMRI study, Canli et al. (2000) showed that pictures rated as "extremely emotionally intense" led to both increased amygdala activation when they were first seen, as well as subsequently better memory than pictures rated as less emotionally intense. This finding points to an explicit role of the amygdala in the enhancement of memory by emotion.

Research in subjects with unilateral amygdala damage has examined memory both for emotionally arousing pictures (Adolphs et al. 2000) and for words (Phelps et al. 1997; LaBar and Phelps 1998). Findings from these studies indicate that damage to the left amygdala results in impaired memory specifically for emotionally arousing pictures and words. In the study by Adolphs et al. (2000), subjects with right amygdala damage showed a normal pattern of enhanced memory for emotional pictures, whereas subjects with left amygdala damage did not show this pattern; however, the study was limited by the small number of subjects with right amygdala damage $(n=2)$. LaBar and Phelps (1998) presented emotionally arousing and neutral words to unilateral temporal lobectomy patients with damage to the amygdala and to normal control subjects. Only the control group showed a differential forgetting rate for the arousing versus neutral words, showing increased delayed recall for the arousing words. Both the right and the left amygdala damaged groups showed decreased memory for the arousing words at delayed recall. Consistent with the Adolphs et

LEARNING \& MEMORY 8:326-335 @ 2001 by Cold Spring Harbor Laboratory Press ISSN1072-0502/01 \$5.00

$$
\begin{array}{lllllllllllllllllllllllll} 
& E & A & R & N & I & N & G & \bigotimes & M & E & M & O & R & Y
\end{array}
$$


al. (2000) study, the left amygdala damaged group showed the poorest memory performance.

Results from these studies are consistent with the material-specific model of memory (Milner 1966; Saykin et al. 1992), which posits that memory function is lateralized with brain function. In short, the medial temporal lobe of the left hemisphere mediates memory for verbal stimuli, whereas the right hemisphere medial temporal lobe structures mediate memory for nonverbal stimuli. This idea has not been tested, however, in the context of the enhancement of declarative memory by emotionally arousing stimuli. If the material-specific principle extends to the enhancement of memory by emotional stimuli, then the lateralized function of the amygdalae may mediate this effect. This study was designed to address this question. Memory performances for emotional pictures and accompanying verbal narratives were assessed in subjects with unilateral temporal lobectomy including damage to the right or left amygdala. We hypothesized that unilateral amygdala damage would impair the normal facilitation of memory by emotion such that (1) emotional memory for verbal material is impaired by left amygdala damage; and (2) emotional memory for nonverbal material is impaired by right amygdala damage.

\section{RESULTS}

\section{Demographic and Neuropsychological Data}

Table 1 shows demographic and neuropsychological test performance data for all groups. There were significant group differences in both age and education. Both groups with amygdala damage were younger than the control groups, although contrasts showed no differences between individual groups in age. The group with right medial temporal lobe damage had a greater extent of damage to both the amygdala and hippocampus, although only the difference in amygdala damage was statistically significant: $Z=2.73, P=0.007$; group difference in hippocampal damage: $Z=1.29, P>0.2$.

There were group differences in neuropsychological test performance on verbal IQ (VIQ), auditory verbal learning test (AVLT), and the controlled oral word association test (COWA). Tukey's post-hoc multiple comparisons revealed no significant differences between individual group

Table 1. Demographic and Neuropsychological Data

\begin{tabular}{|c|c|c|c|c|c|c|}
\hline Demographics & $\mathrm{NC}$ & $\mathrm{BDC}$ & $\mathrm{L}$ amyg & $\mathrm{R}$ amyg & Statistic & $P$ \\
\hline Number of subjects & 36 & 18 & 7 & 7 & NA & NA \\
\hline Age & $47 \pm 7.1$ & $52 \pm 13.6$ & $36 \pm 6.4$ & $28 \pm 7.8$ & $F=13.3$ & $<$ \\
\hline Gender & $17 \mathrm{~F} / 19 \mathrm{M}$ & $11 \mathrm{~F} / 7 \mathrm{M}$ & $4 \mathrm{~F} / 3 \mathrm{M}$ & $3 F / 4 M$ & $x^{2}=1.2$ & $>0.7$ \\
\hline Education & $16 \pm 2.4$ & $13 \pm 2.5$ & $13 \pm 1.6$ & $13 \pm 1.9$ & $F=5.7$ & $0.002 *$ \\
\hline Handedness & 29R/7L & $18 \mathrm{R}$ & $6 \mathrm{R} / 1 \mathrm{~L}$ & $6 \mathrm{R} / 1 \mathrm{~L}$ & $x^{2}=3.9$ & $>0.2$ \\
\hline Amygdala damage & NA & NA & $0.71 \pm 0.3$ & $1.57 \pm 0.5$ & $Z=2.73$ & $0.007^{*}$ \\
\hline Hippocampal damage & NA & NA & $1.23 \pm 0.3$ & $1.67 \pm 0.2$ & $Z=1.29$ & $>0.2$ \\
\hline Age of seizure onset & NA & NA & $5.8 \pm 8.2$ & $8.3 \pm 5.9$ & $Z=0.9$ & $>0.4$ \\
\hline Years post-surgery & NA & NA & $5.2 \pm 5.0$ & $3.1 \pm 2.9$ & $Z=1.25$ & $>0.2$ \\
\hline \multicolumn{7}{|l|}{ Neuropsychological data } \\
\hline VIQ & $102^{* *} \pm 8.9$ & $102 \pm 8.9$ & $91 \pm 13.6$ & $94 \pm 9.1$ & $F=4.5$ & $0.007^{*}$ \\
\hline $\mathrm{PIQ}$ & NA & $101 \pm 9.7$ & $102 \pm 10.3$ & $104 \pm 14$ & $F<1$ & $>0.7$ \\
\hline Benton faces & $46 \pm 3.9$ & $46 \pm 4.3$ & $44 \pm 1.9$ & $46 \pm 2.8$ & $F=1.4$ & $>0.2$ \\
\hline AVLT & $13 \pm 1.9$ & $11 \pm 3.1$ & $9.3 \pm 2.9$ & $11.4 \pm 2.8$ & $F=6.6$ & $0.001^{*}$ \\
\hline VRT correct & $8.0 \pm 1.2$ & $7.3 \pm 1.5$ & $8.3 \pm 1.8$ & $7.1 \pm 1.5$ & $F=1.6$ & 0.19 \\
\hline VRT errors & $2.8 \pm 2.0$ & $4.3 \pm 2.5$ & $2.4 \pm 3.0$ & $4.4 \pm 3.6$ & $F=1.7$ & 0.18 \\
\hline CFT & NA & $19 \pm 7.5$ & $18 \pm 6.9$ & $18 \pm 4.6$ & $F<1$ & $>0.9$ \\
\hline Aphasia & NA & $13 \mathrm{~N} / 4 \mathrm{mi} / 1 \mathrm{mod}$ & $7 \mathrm{~N}$ & $7 \mathrm{~N}$ & $\chi^{2}=4.73$ & 0.094 \\
\hline WCST categories & $5.9 \pm 0.4$ & $5.5 \pm 1.0$ & $5.5 \pm 0.8$ & $5.8 \pm 0.4$ & $F=1.1$ & $>0.3$ \\
\hline COWA & $43 \pm 10.3$ & $30 \pm 9.6$ & $39 \pm 16.0$ & $40 \pm 6.5$ & $F=5.7$ & $0.002 *$ \\
\hline BDI & $4.0 \pm 3.1$ & $5.5 \pm 5.4$ & $5.0 \pm 5.3$ & $5.4 \pm 4.0$ & $F<1$ & $>0.5$ \\
\hline
\end{tabular}

$\mathrm{NC}=$ normal control group; $\mathrm{BDC}=$ brain damaged control group; $\mathrm{L}$ amyg = group with left amygdala damage; $\mathrm{R}$ amyg = group with right amygdala damage. Education is reported in years. Handedness was assessed with the Oldfield-Geschwind Handedness Questionnaire. Amygdala damage on a scale of 0-2 as determined from MRI images; hippocampal damage on a scale of 0-3 as determined from MRI images (see Methods for description of damage assessment). Age of seizure onset in years $(N=13)$; Years post-surgery $=$ time since temporal lobectomy $(\mathrm{N}=13)$. VIQ = WAIS-R Verbal IQ; PIQ = WAIS-R Performance IQ; Benton faces = Benton Facial Recognition Test; $\mathrm{AVLT}=$ Auditory-Verbal Learning Test (number of words recalled on trial 5); VRT = Visual Retention Test, Correct = number correct out of 10, Errors = number of errors committed; CFT = Complex Figure Test, $30 \mathrm{~min}$ recall; Aphasia: subjects classified as $\mathrm{N}=\mathrm{none}, \mathrm{Mi}=$ mild, Mod = moderate; WCST Categories = Wisconsin Card Sort Test number of categories achieved; COWA = Controlled Oral Word Association (number of words generated for the letters C, F, and L); BDI = Beck Depression Inventory.

*Denotes significant main effect of group. Tukey's post-hoc multiple comparisons revealed no significant differences between individual group means. **Estimated verbal IQ based on National Adult Reading Test-Revised (NART-R; Blair and Spreen 1989). Means and standard deviations are shown.

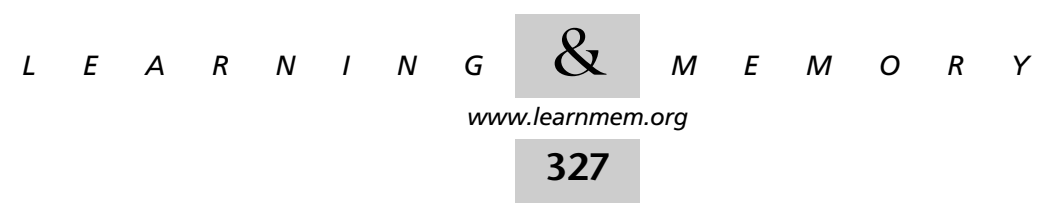


means on these tests. See Table 1 for a full description of these data.

\section{Ratings of Valence and Arousal}

Figure 1 shows the ratings of valence and arousal of each emotional category for each group. Groups did not differ in their ratings of the pictures on either valence $\left(\chi^{2}(3, n=\right.$ $68)=2.8, P=0.4)$ or arousal $\left(\chi^{2}(3, n=68)=5.6, P=0.13\right)$ scales.

\section{Free Recall of Verbal Material}

A comprehensive summary of the statistical findings for the results reported below is given in Table 2 .

Between-subjects analysis showed that the groups differed on overall correct recall of the narratives $\left(\chi^{2}(3\right.$, $n=68)=24.15, P<0.0005)$. Only the group with left amygdala damage was significantly impaired in the recall of unpleasant narratives compared with the normal control group. There were no significant between-groups differ-

\section{A. Valence}
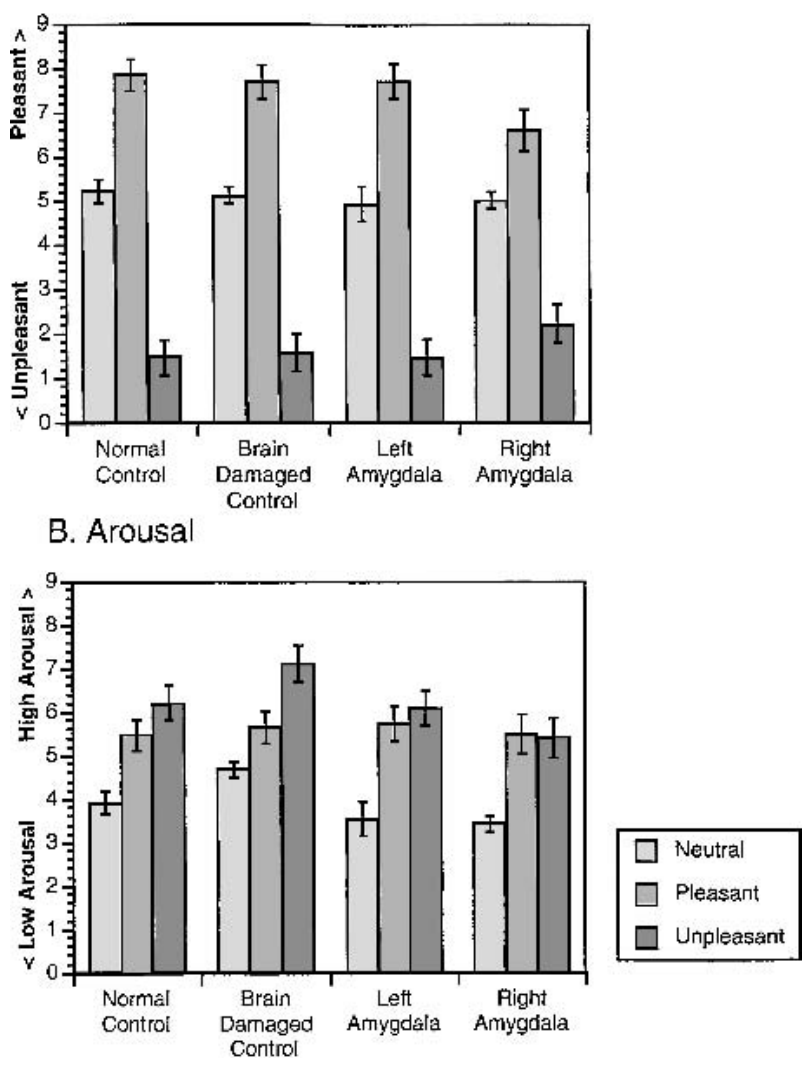

Figure 1 Ratings of picture valence and arousal. (A) Mean ( \pm standard error) ratings of valence (pleasantness) are shown from all subjects across the three emotion categories. The scale represents maximum pleasantness at 9 and maximum unpleasantness at $0 .(B)$ Mean ( \pm standard error) ratings of arousal from all subjects across the three emotion categories. The scale represents maximum arousal at 9 and minimum arousal at 0 . ences in recall for the pleasant or neutral narratives (see Fig. 2 and Table 2).

Within-subjects analysis revealed that for both control groups and the group with right amygdala damage, emotional narratives were recalled better than neutral narratives as assessed by a main effect of narrative emotion on recall (normal controls, $\chi^{2}(2, n=36)=18.05, P<0.0005$; braindamaged controls, $\chi^{2}(2, n=18)=11.09, P=0.0025$; right amygdala group, $\left.\chi^{2}(2, n=7)=6.1, P=0.0408\right)$. The left amygdala damaged group did not show this pattern, however $\left(\chi^{2}(2, n=7)=0.442, P=0.914\right)$. Multiple comparisons revealed that both control groups showed increased memory for the unpleasant narratives compared with both pleasant and neutral narratives. Additionally, the pleasant narratives were better remembered than the neutral narratives by the normal control group. The right amygdala damaged group showed increased memory for unpleasant narratives compared with neutral narratives, but the group with left amygdala damage did not show this pattern. Neither the left nor the right amygdala damaged groups had significantly enhanced memory for the pleasant narratives (see Fig. 2 and Table 2).

\section{Free Recall of Picture Material}

A comprehensive summary of the statistical findings for the results reported below is given in Table 3 .

As in the memory for the verbal materials, there was a significant group difference in memory for the pictures $\left(\chi^{2}\right.$ $(3, n=68)=37.39, P<0.0005)$. Unpleasant pictures were recalled better by the normal control group compared with all three brain-damaged groups ( $Z$ s $>3.1, P$ s $<0.0004)$. The normal control group recalled significantly more pleasant pictures than did the left amygdala damaged group $(Z=2.52, P=0.0052)$. There were no between-groups differences in recall of neutral pictures (see Fig. 2 and Table 3).

Analysis of the pattern of memory for pictures within groups showed that all groups remembered the emotional pictures better than the neutral pictures. Follow-up contrasts illustrated that both control groups and the group with right amygdala damage recalled more unpleasant pictures than neutral pictures $(P \mathrm{~s}<0.008)$. The group with left amygdala damage showed a trend toward increased recall of the unpleasant compared with the neutral pictures, but this effect was not statistically significant at the conservative $Z=2.128$ level. Additionally, both control groups remembered the pleasant pictures better than the neutral pictures. Neither amygdala damaged group had significantly better recall of pleasant compared with neutral pictures.

\section{Arousal and Free Recall}

We followed up these findings by directly examining the role of arousal on free recall performance, independent of the role of valence (See Fig. 3). Stimuli were divided into a low-arousal and a high-arousal group by median split on the

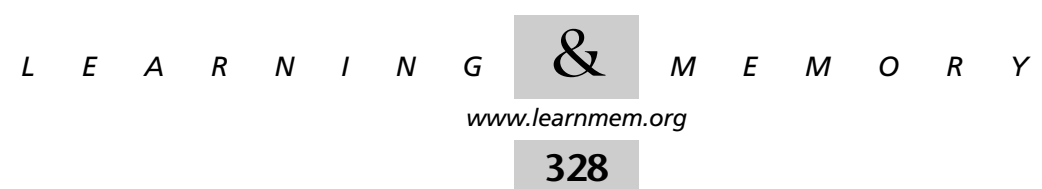


Table 2. Summary of Results for Free Recall of Narratives

\begin{tabular}{|c|c|c|}
\hline Analysis of narrative free recall & Statistic & $P$-value \\
\hline \multicolumn{3}{|l|}{ Between-groups differences } \\
\hline Overall group difference $(N=68)$ & $\chi^{2}=24.15$ & $<0.0005^{*}$ \\
\hline Unpleasant: Normal control vs. Left amygdala & $Z=2.61$ & $0.0036^{* *}$ \\
\hline \multicolumn{3}{|l|}{ Within-group effects of emotion category } \\
\hline Normal controls $(N=36)$ & $\chi^{2}=18.05$ & $0.0005^{*}$ \\
\hline Unpleasant vs. Pleasant & $Z=2.833$ & $0.0018+$ \\
\hline Unpleasant vs. Neutral & $Z=4.01$ & $<0.0001+$ \\
\hline Pleasant vs. Neutral & $Z=1.967$ & 0.0255 \\
\hline Brain damaged controls $(N=18)$ & $\chi^{2}=11.09$ & $0.0025^{*}$ \\
\hline Unpleasant vs. Pleasant & $Z=2.829$ & $0.0017+$ \\
\hline Unpleasant vs. Neutral & $Z=3.204$ & $0.0003+$ \\
\hline Pleasant vs. Neutral & $Z=1.186$ & 0.1875 \\
\hline Left amygdala damage $(N=7)$ & $x^{2}=0.442$ & 0.914 \\
\hline Unpleasant vs. Pleasant & $\hat{Z}=0.0$ & 0.75 \\
\hline Unpleasant vs. Neutral & $Z=1.414$ & 0.25 \\
\hline Pleasant vs. Neutral & $Z=1.0$ & 0.5 \\
\hline Right amygdala damage $(N=7)$ & $\chi^{2}=6.098$ & $0.0408 *$ \\
\hline Unpleasant vs. Pleasant & $Z=1.725$ & 0.0781 \\
\hline Unpleasant vs. Neutral & $Z=2.264$ & $0.0156+$ \\
\hline Pleasant vs. Neutral & $Z=0.817$ & 0.375 \\
\hline
\end{tabular}

average ratings given by all subjects. The median of arousal for the 15 stimuli across all groups was five. This split resulted in eight stimuli classified as high-arousal (three pleasant and all five unpleasant) and seven stimuli classified as low-arousal (two pleasant and all five neutral). Proportions of correct recall of high- versus low-arousal stimuli were used for analysis. The normal control group recalled the high-arousal narratives and pictures significantly better than did all brain-damaged groups $(Z s>2.394, P$ s $<0.007)$. The high-arousal pictures were better remembered by the normal control group compared with all groups with brain damage $(Z s>2.4, P s<0.006)$, but recall of the low-arousal narratives by the normal control group was only significantly better when compared with the brain-damaged control group $(Z=2.448, P=0.0064)$ and not when compared with the groups with amygdala damage.

Within-group analysis comparing recall of high- versus low-arousal narratives revealed that both control groups and the group with right amygdala damage recalled highly arousing narratives better than low-arousal narratives (normal control group: $Z=3.86, P<0.00001$; brain-damaged control group: $Z=2.738, P=0.021$; right amygdala damaged group: $Z=2.214, P=0.0156$ ). The group with left amygdala damage did not show this pattern, however $(Z=1.342, P=0.25)$. For the picture stimuli, both control groups as well as the group with right amygdala damage recalled more from the high-arousal compared with the low-arousal category (normal control group: $Z=4.494$, $P<0.00001 ;$ brain-damaged control group: $Z=3.244$, $P=0.0002$; right amygdala damaged group: $Z=2.375$,
$P=0.0078)$. The group with left amygdala damage did not show this pattern $(Z=1.357, P=0.1172)$. These data indicate a highly significant effect of emotional arousal for memory in a normal control population that is diminished following brain damage and specifically following damage to the left amygdala.

Correlations of Stimulus Ratings and Free Recall A further analysis of the individual stimuli, rank ordered according to the mean arousal ratings that they received, showed that in only the normal control group was the perceived arousal of the stimulus correlated with recall for both narratives $\left(r_{\mathrm{s}}=0.57, \quad P=0.0168\right)$ and pictures $\left(r_{\mathrm{s}}=0.77, P=0.0004\right)$. None of the groups with brain damage showed this pattern, however $\left(r_{\mathrm{s}} \mathrm{s}<0.4, P \mathrm{~s}>0.2\right)$. There was no relationship between perceived pleasantness of the stimuli and free recall performance for any of the groups $\left(r_{\mathrm{s}} \mathrm{s}<0.4, P \mathrm{~s}>0.065\right)$. These data confirm a robust effect of emotional arousal on memory in a normal control population.

\section{Gender Differences}

Difference in recall performance between men and women was assessed between and within groups. Between-group analysis revealed that there was not an overall effect of gender on recall of either narratives $(Z=0.85, P>0.3)$ or pictures $(Z=1.76, \quad P=0.078)$, although the women showed slightly better performance than the men did. Within-group analysis revealed that among the normal control subjects, the women recalled more narratives $(Z=2.1$,

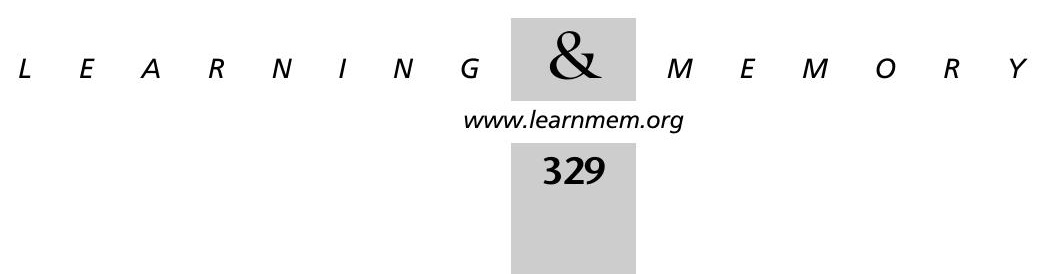


A. Narrative Free Recall by Valence

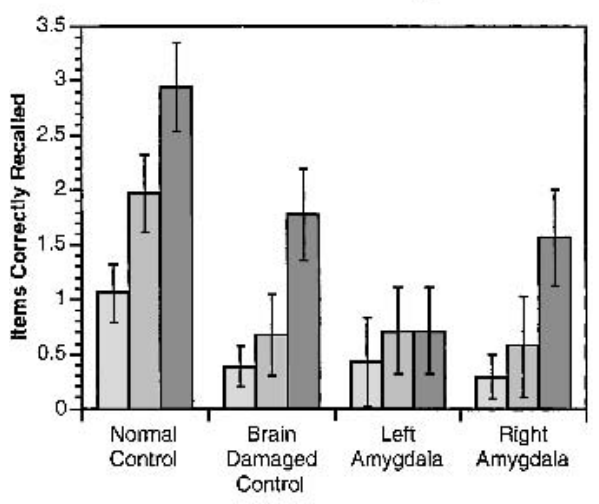

B. Picture Free Recall by Valence

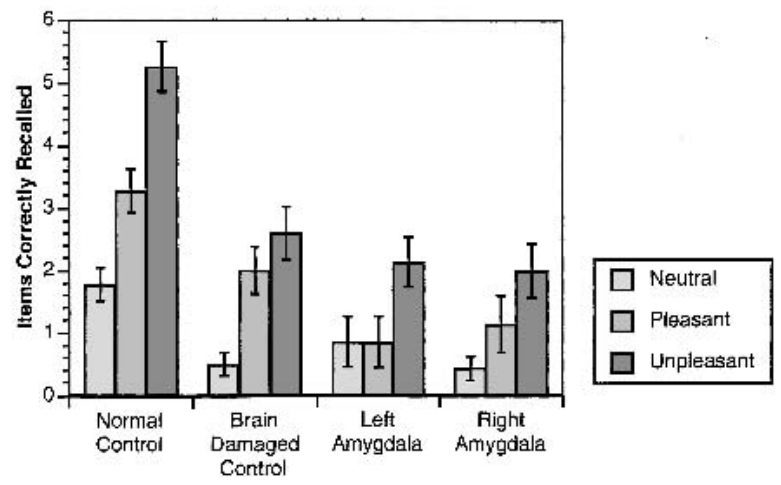

Figure 2 Free recall for narratives and pictures across valence categories. (A) Mean ( \pm standard error) of correctly recalled narratives across all subjects from each valence category. (B) Mean ( \pm standard error) of correctly recalled pictures across all subjects from each valence category.

$P=0.035)$ and pictures $(Z=2.8, P=0.004)$ than did men. Follow-up contrasts between individual valence categories showed that women recalled significantly more pleasant stimuli than men did (narratives: $Z=2.26, P=0.026$; pictures: $Z=2.3, P=0.021$ ). In none of the groups with brain damage was there a gender difference in recall of narratives or pictures $(P \mathrm{~s}>0.11)$.

\section{DISCUSSION}

Results from this study provide further support for the modulatory role of the human amygdala in the enhancement of memory by emotionally salient stimuli. Subjects with left medial temporal lobe damage including the amygdala failed to show the robust enhancement of memory for emotionally arousing verbal stimuli that subjects with right medial temporal lobe damage including the amygdala, normal controls, and brain-damaged controls exhibited. Subjects with right amygdala damage, on the other hand, did show enhanced memory for both verbal and nonverbal emotional stimuli, although their overall memory performance was reduced compared with controls. Therefore, only the first of our original hypotheses was supported, in that the left amygdala damaged group showed disproportionate impairment of memory for emotional verbal stimuli. The right amygdala damaged group, however, was not specifically impaired on memory for emotional nonverbal stimuli. These findings corroborate previous research showing a more pronounced verbal memory deficit following damage to the left versus the right medial temporal lobe and partially support the material-specific model of memory in the context of the enhancement of memory by emotional stimuli.

Previous research on emotional memory in subjects with unilateral amygdala damage has shown that left amygdala damage results in a greater impairment specific to emotional memory than does comparable damage on the right (Phelps et al. 1997; LaBar and Phelps 1998; Adolphs et al. 2000). The authors of these studies have proposed that this specific emotional memory impairment may be attributable to the verbal nature of the tasks in question, in light of the known role of the left temporal lobe in verbal memory (see Milner 1971; Blake et al. 2000). Whereas only two of these studies focused on memory for emotional words (Phelps et al. 1997; LaBar and Phelps 1998), the third used a combination of nonverbal pictures and verbal narratives as stimuli (Adolphs et al. 2000). Results from the current investigation illustrate that damage to the left amygdala impairs verbal emotional memory to a greater extent than nonverbal emotional memory. Although the left amygdala group showed no enhancement of memory for the emotional narratives, their memory for unpleasant pictures was greater than their memory for both neutral and pleasant pictures. This finding illustrates that the disruption of the enhancement of memory for emotional stimuli following amygdala damage is to some extent material-specific.

Recent research examining the material-specific model of memory in brain-damaged patients has shown that damage to both the left and right medial temporal lobes results in verbal memory impairment, although to a greater extent, following left-sided damage (Dobbins et al. 1998). Dobbins and colleagues suggest that this pattern be referred to as "material related" rather than "material specific" to highlight the bilateral involvement of the medial temporal lobes in the formation of verbal memories (Dobbins et al. 1998). Recent work has indicated a reappraisal of the materialspecific model in terms of the different phases of memory (acquisition, recall, and retention; Vanderploeg et al. 2001) as well as in the strict lateralization of verbal versus nonverbal memory performance (Dobbins et al. 1998). Results from the current study could best be characterized as supporting a material-related model of the enhancement of memory by emotional arousal as both groups with amygdala damage were impaired in memory performance, but only the group with left-sided damage showed the predicted pattern of results.

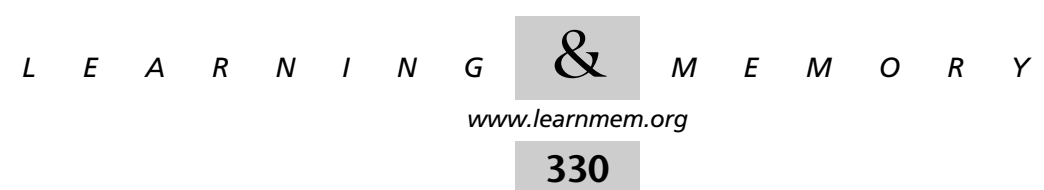


Table 3. Summary of Results for Free Recall of Pictures

\begin{tabular}{|c|c|c|}
\hline Analysis of picture free recall & Statistic & $P$-value \\
\hline \multicolumn{3}{|l|}{ Between-groups differences } \\
\hline Overall group difference $(N=68)$ & $\chi^{2}=37.39$ & $<0.0005^{*}$ \\
\hline Unpleasant: Normal control vs. Brain damaged & $Z=3.771$ & $<0.0001^{* *}$ \\
\hline Unpleasant: Normal control vs. Left amygdala & $Z=3.18$ & $0.0003^{* *}$ \\
\hline Unpleasant: Normal control vs. Right amygdala & $Z=3.246$ & $0.0003^{* *}$ \\
\hline Pleasant: Normal control vs. Left amygdala & $Z=2.52$ & $0.0052^{* *}$ \\
\hline \multicolumn{3}{|l|}{ Within-group effects of emotion category } \\
\hline Normal controls $(N=36)$ & $\chi^{2}=36.82$ & $<0.0005^{*}$ \\
\hline Unpleasant vs. Pleasant & $Z=3.855$ & $<0.0001 \dagger$ \\
\hline Unpleasant vs. Neutral & $Z=4.977$ & $<0.0001+$ \\
\hline Pleasant vs. Neutral & $Z=3.074$ & $0.0007+$ \\
\hline Brain damaged controls $(N=18)$ & $\chi^{2}=17.04$ & $<0.0005^{*}$ \\
\hline Unpleasant vs. Pleasant & $Z=2.183$ & 0.0168 \\
\hline Unpleasant vs. Neutral & $Z=3.557$ & $<0.0001+$ \\
\hline Pleasant vs. Neutral & $Z=3.213$ & $0.0001+$ \\
\hline Left amygdala damage $(N=7)$ & $\chi^{2}=6.212$ & $0.0394^{*}$ \\
\hline Unpleasant vs. Pleasant & $Z=1.807$ & 0.0625 \\
\hline Unpleasant vs. Neutral & $Z=1.725$ & 0.0781 \\
\hline Pleasant vs. Neutral & $Z=1.374$ & 0.5 \\
\hline Right amygdala damage $(N=7)$ & $x^{2}=4.68$ & $0.0312 *$ \\
\hline Unpleasant vs. Pleasant & $Z=1.561$ & 0.0859 \\
\hline Unpleasant vs. Neutral & $Z=2.46$ & $0.0078+$ \\
\hline Pleasant vs. Neutral & $Z=1.394$ & 0.1094 \\
\hline
\end{tabular}

The group with damage to the left amygdala had somewhat lower verbal IQ and AVLT scores compared with the other brain-damaged groups. Post-hoc comparisons, however, did not show specific differences in VIQ nor AVLT performance between the two groups with amygdala damage. Correlation analyses between VIQ, AVLT, and memory performance indicated that these variables were unrelated to recall performance on these tasks. Even in light of a pre-existing verbal memory deficit (as indexed by the AVLT), recall of neutral stimuli by the group with left amygdala damage was identical to that of both the brain-damaged controls and the group with right amygdala damage. The only difference among these groups' performance was in recall of emotionally arousing stimuli, indicating that left medial temporal lobe damage including the amygdala in these subjects mainly affected the facilitation of memory by emotional arousal and is not indicative of a generalized memory deficit.

Performance by the group with right amygdala damage was more similar to the control groups than was the performance of the left amygdala damaged group. The hypothesized deficit in recall of nonverbal compared with verbal stimuli following right amygdala damage was not supported by the results. Several studies have documented deficits in visuospatial processing and memory for complex visual scenes following right temporal lobectomy (Barr 1997; Glosser et al. 1998; Pillon et al. 1999). One possible mecha- nism that might account for the impaired verbal emotional memory in subjects with left amygdala damage, on the one hand, and yet the preserved nonverbal emotional memory in subjects with right amygdala damage, on the other hand, is the following. Memory for visual stimuli in the current study may have been recoded into verbal information, whereas it may be more difficult to perform the converse operation and recode verbal memory into a visual format. Further, this effect could have been prompted by the fact that the memory tests explicitly called for verbal-format responses. Results from the group with right amygdala damage do not support a material-specific model of memory performance.

\section{Arousal and Valence of the Stimuli}

Both groups with amygdala damage rated the picture stimuli as similarly arousing and pleasant as did the control groups. Specifically, across all groups, the a priori emotion categories of pleasant, unpleasant, and neutral were given the expected ratings, with the unpleasant stimuli rated the least pleasant and the most arousing, whereas the pleasant slides were rated the most pleasant and more arousing than the neutral slides. The unpleasant pictures were remembered better by both amygdala damaged groups (although this enhancement was statistically significant only in the right amygdala damaged group). This difference in performance among the groups is most likely caused by the dif-

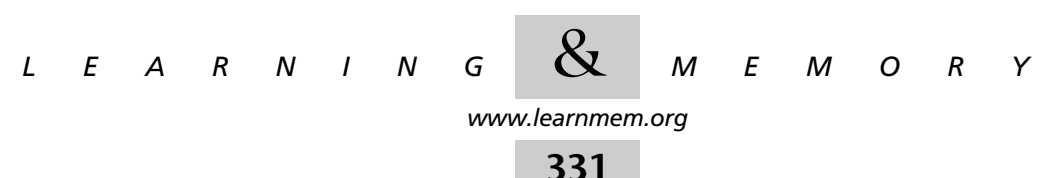




\section{A. Narrative Free Recall by Arousal}

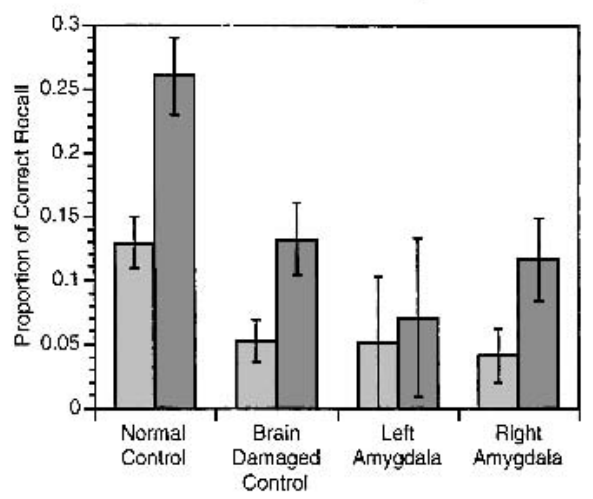

B. Picture Free Recall by Arousal

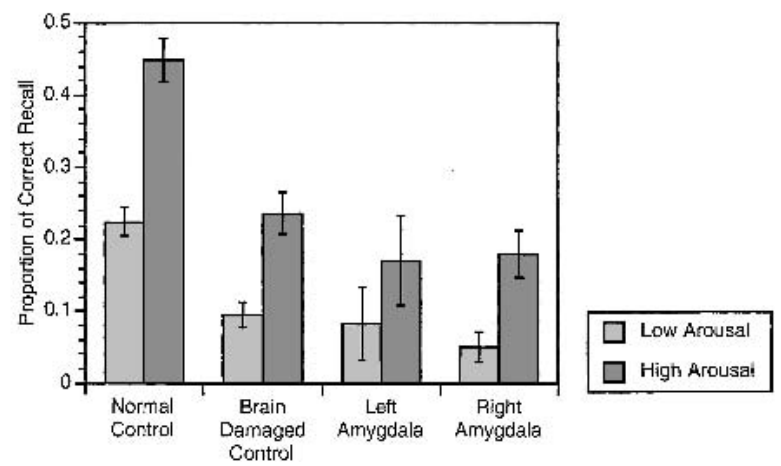

Figure 3 Free recall for narratives and pictures across arousal categories. (A) Mean ( \pm standard error) of the proportion of correctly recalled narratives across all subjects from low and high arousal categories. (B) Mean ( \pm standard error) of the proportion of correctly recalled pictures across all subjects from low- and high-arousal categories.

ferences in the arousing nature of the pleasant versus the unpleasant pictures. Numerous studies of emotional memory have illustrated that the arousing nature of the to-be-remembered stimuli is more predictive of subsequent memory performance than ratings of pleasantness (Bradley et al. 1992; Bradley and Lang 2000). Considerable research has indicated a role for the amygdala in the establishment of memory for highly arousing unpleasant (Cahill et al. 1996; Adolphs et al. 1997, 2000) and pleasant (Hamann et al. 1999) visual stimuli. Perhaps the arousal level of the pleasant stimuli that resulted in increased recall performance in the control groups was not enough to influence memory in the unilateral amygdala damaged groups. Classification of these stimuli by perceived arousal level illustrates that for the normal control group, higher perceived arousal, but not pleasantness, was correlated with greater recall performance. Valence was not correlated with memory performance in any of the groups. This result further implicates emotional arousal, over and above pleasantness, as the crucial factor in the enhancement of memory by emotion. Future work could more explicitly examine the relationships among arousal, pleasantness, and the amygdala in the enhancement of memory by using stimuli of differing valence that are precisely matched in terms of their arousal.

\section{Relation to Lesion Extent}

The marked discrepancy between the performance of subjects with left amygdala damage versus those with right amygdala damage is surprising in light of the differences between the groups in the extent of amygdala and hippocampal damage. The group with right amygdala damage actually had a somewhat greater extent of damage to both the amygdala and hippocampus (although the differences in hippocampal damage between the groups were not statistically significant) than the group with left-sided damage, and yet the right group showed better overall memory performance and a more normal pattern of emotional facilitation of memory than did the left group. These data indicate that a smaller extent of damage to the left medial temporal lobe than right medial temporal lobe is sufficient to produce impaired memory performance. Additionally, data from these subjects indicate that a smaller extent of damage to the left compared with the right amygdala is sufficient to produce a deficit in the enhancement of memory by emotionally arousing stimuli. This finding lends further support to the idea that the left amygdala is disproportionately important for the enhancement of declarative memory by emotion.

\section{Gender Differences}

Recent neuroimaging research has indicated a gender difference in the pattern of the influence of the amygdala in the enhancement of memory by emotional arousal (Canli et al. 2000; Cahill et al. 2001). Results from these studies have shown that activity in the right amygdala of men and the left amygdala of women was related to enhanced memory for emotional material. Analysis of gender differences in the current experiment illustrated that there were gender differences in memory performance, but these differences were only found in the normal control participants. Women in the normal control group recalled significantly more information than did men. There was no gender difference in performance in any of the patient groups, nor was there an interaction between gender and side of lesion related to memory performance. Although the groups were roughly equivalent in the number of men and women in each group, we did not expect any gender differences in relation to performance on this task in relation to the laterality of lesion. Additionally, the small sample size of the patient groups may have precluded the observation of gender differences.

\section{The Amygdala's Role in Memory Consolidation} In light of the established role of the medial temporal lobe memory system - which includes the hippocampus and ad-

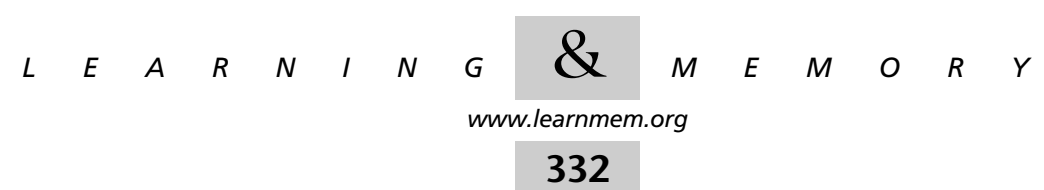


jacent cortex (Cohen and Eichenbaum 1993)-how does the amygdala fit in? The detection and experience of emotion increases the processing of emotional stimuli by focusing attention and enacting response systems (Frijda 1987; Lang et al. 1990). The amygdala has an integral role in this enhanced processing through influence over numerous brain structures including the hippocampus, hypothalamus, striatum, brain stem arousal centers, as well as bidirectional connections with neocortex (Amaral et al. 1992). One effect of experienced arousal and amygdala activation is the enhancement of declarative memories for situations surrounding the arousing stimuli. Animal research indicates a mechanism for this effect by showing that stimulation of the basolateral amygdala enhances long-term potentiation (LTP) in both the dentate gyrus (Ikegaya et al. 1995) and insular cortex (Escobar et al. 1998). In addition to these direct effects of amygdala activity, the memory-enhancing effects of stress hormones are dependent on an intact amygdala (McGaugh et al. 1996). The final common pathway of the influence of these hormones appears to be $\beta$-adrenergic activity within the amygdala, as blockade of amygdaloid $\beta$-adrenergic receptors blocks the enhancement of memory by emotion (McGaugh et al. 1988). Recent work documenting an increase in memory for emotional stimuli following administration of the human stress hormone cortisol has indicated an extension of these findings to humans (Buchanan and Lovallo 2001). Human neuroimaging results from Hamann and colleagues (1999) extend this notion by showing that both amygdala and hippocampal activity during the encoding of emotionally arousing material was correlated with subsequent enhanced memory of this material. These findings place the amygdala in a modulatory position of the traditional medial temporal lobe memory system, its activity influencing memory formation under conditions of emotional arousal.

\section{MATERIALS AND METHODS}

\section{Subjects}

Fourteen subjects with unilateral medial temporal lobe damage (seven left and seven right) and 18 brain-damaged controls with lesions sparing the amygdala and hippocampus were selected from the Patient Registry of the Division of Behavioral Neurology and Cognitive Neuroscience at the University of Iowa. Thirteen of the 14 subjects with unilateral amygdala damage had undergone temporal lobectomy for the treatment of epilepsy. One member of the group with left amygdala damage received damage caused by a closed-head injury affecting the amygdala, hippocampus, as well as temporal polar cortex. Additionally, 36 normal subjects were recruited through local advertisement. All participants were individually administered a 2- to 3-h neuropsychological battery that included measures of intellect, anterograde verbal and visual memory, visuoperception, language, and executive functioning (Tranel 1996). All subjects gave informed consent to participate in these studies, which were approved by the Human Subjects Committee of the University of Iowa.
Table 1 lists the extent of amygdala and hippocampus damage along with demographic information and neuropsychological performance data for all groups. All brain-damaged subjects underwent an MR scan (except for one subject who had a CT scan) and the neuroanatomy of each lesion was determined from detailed threedimensional reconstructions of their brains from the scans (Damasio and Frank 1992). We included only subjects with single, focal, stable lesions. Assessment of the extent of amygdala and hippocampus damage was conducted individually for each subject in the temporal lobe damaged groups (except for one subject who only had CT scans). Each subject's brain was aligned according to the coordinate system of Talairach and Tournoux (Talairach and Tournoux 1988) using the anterior and posterior commisures as guides. Following alignment, the boundaries of both the amygdala and hippocampus were ascertained. Criteria for the boundaries of both the amygdala and hippocampus were derived from the atlas of Duvernoy (Duvernoy 1988).

The amygdala boundaries were defined anteriorly using the white matter of the parahippocampal gyrus as the anterior, lateral and inferior borders. Posteriorly, the amygdala was bounded by the temporal horn of the lateral ventricle as the inferior border, the basal ganglia as the superior border, the uncus as the medial border, the white matter of the temporal lobe as the lateral border, and the hippocampus as the posterior border. Assessment of amygdala damage was accomplished by examination of the area defined by these borders in comparison with the contralateral amygdala on coronal slices. Scores of 0,1 , or 2 were assigned to each coronal slice passing through the amygdala based on the amount of damage observed within that slice. A score of 0 was given if there was no discernible damage, 1 if $<50 \%$ of the area was damaged, and 2 if $>50 \%$ of the area was damaged. The mean value of these ratings across each coronal slice through the amygdala served as the index of amygdala damage.

The boundaries of the hippocampus were defined anteriorly by the amygdala, posteriorly by the fornix and splenium of the corpus callosum, and inferiorly, superiorly, and laterally by the white matter of the parahippocampal gyrus and the temporal horn of the lateral ventricle. Hippocampal damage assessment was accomplished in a similar manner to that described for the amygdala. Scores of $0,1,2$, or 3 were assigned to each coronal slice passing through the hippocampus based on the amount of damage observed within that slice. A score of 0 was given if there was no discernible damage, 1 if $<25 \%$ of the area was damaged, 2 if between $25 \%$ and $75 \%$ of the area was damaged, and 3 if $>75 \%$ of the area was damaged. The mean value of these ratings across each coronal slice through the hippocampus served as the index of hippocampal damage. Expert raters trained in neuroanatomical analysis completed neuroanatomical ratings. The raters were blind as to the identity of each subject, although the group membership was obvious from the side of the lesion.

\section{Tasks}

All subjects were tested individually on two consecutive days. On the first day, subjects were shown 15 color photographs. The stimuli consisted of five pleasant (e.g., puppies), five unpleasant (e.g., mutilated bodies), and five neutral (e.g., a classroom setting) pictures. Several of the pictures were chosen from the International Affective Picture System (CSEA-NIMH 1995), whereas the rest were drawn from print-media sources. All stimuli included depictions of people and were chosen to be of approximately equal visual complexity.

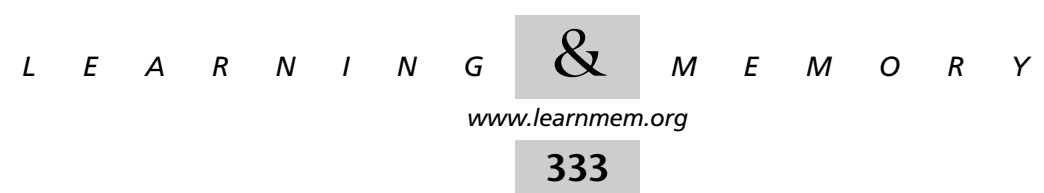


Subjects were seated in a dark room while watching the stimuli and had electrodes placed on each palm for the measurement of skin conductance responses (to be reported elsewhere). Each stimulus was presented for 20 seconds on a computer monitor and was accompanied by a simple, single-sentence narrative description read by one of the experimenters. An example of the narrative description for a photograph of two young girls eating ice cream read as follows: Lizzie and Susie loved to eat vanilla ice cream together. Each narrative sentence contained information that could not have been obtained from the picture. For example in the picture of girls eating ice cream, the names of the girls were given only in the narrative. Subjects were told to watch the stimuli and listen to the spoken narrative for the expressed purpose of monitoring their psychophysiological response to visual and auditory stimuli. No mention of a memory test was made; encoding of the stimuli was therefore incidental. Stimuli were shown in three blocks of five stimuli each, with stimuli in each block presented in a counterbalanced order. Between each stimulus within a block, there was a 20-sec interstimulus interval of a blank screen. Blocks were separated by a 1 -min break.

Twenty-four hours after the encoding session, subjects returned to the laboratory and participated in a free recall test in which they were told to write down as much information as they could recall about each picture that they had seen and, separately, each narrative that they had heard. There was no time limit for the memory testing. A trained technician who was blind to each subject's group membership then scored subjects' written responses according to standardized criteria. Responses were coded as " 0 " if there was no information linking the response to any of the stimuli, "1" if a response could be unambiguously linked to a particular narrative or picture but omitted or was in error about some of the information, and " 2 " if a response could definitely be linked to a specific narrative or picture and stated correct details. Following free recall testing, subjects were shown the pictures again and were asked to rate them on separate nine-point Likert scales of valence (pleasantness) and arousal.

\section{Data Analysis}

Because of the large differences in group sizes, the small group sizes of the amygdala damaged groups, and the non-normality of the distribution of the data, nonparametric statistics accompanied by exact $P$-values or Monte Carlo estimates of exact $P$-values were used throughout the analysis of these data, as suggested by Siegel and Castellan (1988). Such exact $P$-values are obtained by re-randomizations of the data, to generate the permutation distribution for the data (or an estimate thereof). Exact probabilities computed in this way do not make any of the underlying assumptions of inferential statistics; in particular, they do not assume a shape of the underlying population distribution from which the sample was drawn and are therefore more conservative indicators of whether or not the null hypothesis should be rejected.

\section{Between-Subjects Analysis}

Data were first analyzed across all four groups using a KruskalWallis one-way analysis of variance (ANOVA) by ranks to test for the main effect of group membership on all variables. Results from this test are reported as the value based on the $\chi^{2}$ distribution with 3 degrees of freedom and sample size of $68\left[\chi^{2}(3, n=68)\right]$. Monte Carlo estimates of the exact $P$-values were computed for significance testing of this test. Multiple comparisons were then conducted using Wilcoxon-Mann-Whitney tests and exact $P$-values to determine group differences across each emotion category. Results from these analyses are reported using the $Z$-scored difference between means. These comparisons were adjusted to avoid inflation of the Type I error rate by comparing the $Z$-scored group differences against the critical $Z$ values for six multiple comparisons $(Z=2.394$, one-tailed; Siegel and Castellan 1988).

\section{Within-Subjects Analysis}

Separate Kruskal-Wallis one-way ANOVAs were conducted within each subject group to examine differences in the pattern of memory performance within each group across emotion categories. Results from this test are reported as the value based on the $\chi^{2}$ distribution with 2 degrees of freedom; sample size varies by group and is reported for individual tests. Finally, multiple comparisons were conducted individually for each group using pairwise Wilcoxon Signed Ranks tests of recall for the different emotion categories (pleasant vs. unpleasant, pleasant vs. neutral, unpleasant vs. neutral). These analyses were corrected for multiple comparisons by comparing the $Z$-scored difference between emotion categories against the critical $Z$ values for three multiple comparisons $(Z=2.128$, one-tailed; Siegel and Castellan 1988). All analyses were conducted using StatXact 4 software (Cytel Software Corporation), which provides routines for computing exact probabilities using re-randomization methods

\section{Correlation Analysis}

Measure of association between average rating of arousal for each stimulus (across all subjects) and average free recall performance within each subject was conducted using Spearman's rank-order correlation coefficient along with exact $P$-values. These analyses were computed separately for each group.

\section{ACKNOWLEDGMENTS}

The authors thank Matt Karafin for assistance in scoring memory tests, Ken Manzel for assistance with testing and obtaining information about subjects' medical status, and Denise Krutzfeldt for help in scheduling subjects' visits. Additionally, we thank Drs. John Allen and Hanna Damasio for neuroanatomical guidance. This study was supported by a grant from NIMH to R.A. (R29 MH57905-03) and by Program Project Grant NINDS NS19632.

The publication costs of this article were defrayed in part by payment of page charges. This article must therefore be hereby marked "advertisement" in accordance with 18 USC section 1734 solely to indicate this fact.

\section{REFERENCES}

Adolphs, R., Cahill, L., Schul, R., and Babinsky, R. 1997. Impaired declarative memory for emotional material following bilateral amygdala damage in humans. Learn. Mem. 4: 291-300.

Adolphs, R., Tranel, D., and Denburg, N. 2000. Impaired emotional declarative memory following unilateral amygdala damage. Learn. Mem. 7: 180-186.

Amaral, D.G., Price, J.L., Pitkanen, A., and Carmichael, S.T. 1992. Anatomical organization of the primate amygdaloid complex. In The amygdala: Neurobiological aspects of emotion, memory, and mental dysfunction (ed. J.P. Aggleton), pp. 1-66. Wiley-Liss, NY.

Barr, W.B. 1997. Examining the right temporal lobe's role in nonverbal memory. Brain Cogn. 35: 26-41

Blair, J.R. and Spreen, O. 1989. Predicting premorbid IQ: A revision of the national adult reading test. Clin. Neuropsychol. 3: 129-136.

Blake, R.V., Wroe, S.J., Breen, E.K., and McCarthy, R.A. 2000. Accelerated forgetting in patients with epilepsy: Evidence for an impairment in memory consolidation. Brain 123: 472-483. 
Bradley, M.M. and Lang, P.J. 2000. Affective reactions to acoustic stimuli. Psychophysiology 37: 204-215.

Bradley, M.M., Greenwald, M.K., Petry, M.C., and Lang, P.J. 1992. Remembering pictures: Pleasure and arousal in memory. J. Exp. Psychol. Learn. Mem. Cogn. 18: 379-390.

Buchanan, T.W. and Lovallo, W.R. 2001. Enhanced memory for emotional material following stress-level cortisol treatment in humans. Psychoneuroendocrinology 26: 307-317.

Cahill, L., Babinsky, R., Markowitsch, H.J., and McGaugh, J.L. 1995. The amygdala and emotional memory. Nature 377: 295-296.

Cahill, L., Haier, R.J., Fallon, J., Alkire, M.T., Tang, C., Keator, D., Wu, J., and McGaugh, J.L. 1996. Amygdala activity at encoding correlated with long-term, free recall of emotional information. Proc. Natl. Acad. Sci. 93: 8016-8021.

Cahill, L., Haier, R.J., White, N.S., Fallon, J., Kilpatrick, L., Lawrence, C., Potkin, S.G., and Alkire, M.T. 2001. Sex-related difference in amygdala activity during emotionally influenced memory storage. Neurobiol. Learn. Mem. 75: 1-9.

Canli, T., Zhao, Z., Brewer, J., Gabrieli, J.D., and Cahill, L. 2000. Event-related activation in the human amygdala associates with later memory for individual emotional experience. J. Neurosci. 20: RC99.

Cohen, N. J. and Eichenbaum, H. 1993. Memory, amnesia, and the hippocampal system. MIT Press, Cambridge, MA.

Center for the Study of Emotion and Attention (CSEA-NIMH). 1995. The international affective picture system: Photographic slides. The Center for Research in Psychophysiology, University of Florida, Gainesville, FL.

Damasio, H. and Frank, R. 1992. Three-dimensional in vivo mapping of brain lesions in humans. Arch. Neurol. 49: 137-143.

Dobbins, I.G., Kroll, N.E., Tulving, E., Knight, R.T., and Gazzaniga, M.S. 1998. Unilateral medial temporal lobe memory impairment: type deficit, function deficit, or both? Neuropsychologia 36: 115-127.

Duvernoy, H.M. 1988. The buman bippocampus: An atlas of applied anatomy. Springer-Verlag, NY.

Escobar, M.L., Chao, V., and Bermudez-Rattoni, F. 1998. In vivo long-term potentiation in the insular cortex: NMDA receptor dependence. Brain Res. 779: 314-319.

Frijda, N.H. 1987. Emotion, cognitive structure, and action tendency. Cognit. Emotion 1: 115-143.

Glosser, G., Deutsch, G.K., Cole, L.C., Corwin, J., and Saykin, A.J. 1998 Differential lateralization of memory discrimination and response bias in temporal lobe epilepsy patients. J. Int. Neuropsychol. Soc. 4: 502-511.

Hamann, S.B., Ely, T.D., Grafton, S.T., and Kilts, C.D. 1999. Amygdala activity related to enhanced memory for pleasant and aversive stimuli. Nat. Neurosci. 2: 289-293.

Heuer, F., and Reisberg, D. 1990. Vivid memories of emotional events: The accuracy of remembered minutiae. Mem. Cognit. 18: 496-506.

Ikegaya, Y., Saito, H., and Abe, K. 1995. Requirement of basolateral amygdala neuron activity for the induction of long-term potentiation in the dentate gyrus in vivo. Brain Res. 671: 351-354.

LaBar, K.S. and Phelps, E.A. 1998. Arousal-mediated memory consolidation: Role of the medial temporal lobe in humans. Psychological Sci. 9: 490-493.

Lang, P.J., Bradley, M.M., and Cuthbert, B.N. 1990. Emotion, attention, and the startle reflex. Psychol. Rev. 97: 377-395.

McGaugh, J.L. 2000. Memory-a century of consolidation. Science 287: $248-251$.
McGaugh, J. L. and Cahill, L. 1997. Interaction of neuromodulatory systems in modulating memory storage. Behav. Brain Res. 83: 31-38.

McGaugh, J.L., Introini-Collison, I.B., and Nagahara, A.H. 1988. Memory-enhancing effects of posttraining naloxone: Involvement of beta-noradrenergic influences in the amygdaloid complex. Brain Res. 446: 37-49.

McGaugh, J.L., Introini-Collison, I.B., Nagahara, A.H., Cahill, L., Brioni, J.D., and Castellano, C. 1990. Involvement of the amygdaloid complex in neuromodulatory influences on memory storage. Neurosci. Biobehav. Rev. 14: 425-431.

McGaugh, J.L., Cahill, L., and Roozendaal, B. 1996. Involvement of the amygdala in memory storage: interaction with other brain systems. Proc. Natl. Acad. Sci. 93: 13508-13514.

Milner, B. 1966. Amnesia following operation on the temporal lobes. In Amnesia. (ed. C.W.M. Whitty and O.L. Zangwill), pp. 109-133. Butterworths, London, UK.

. 1971. Interhemispheric differences in the localization and psychological processes in man. Brit. Med. Bull. 27: 272-277.

Murray, E.A. 1992. Medial temporal lobe structures contributing to recognition memory: The amygdaloid complex versus the rhinal cortex. In The amygdala: Neurobiological aspects of emotion, memory, and mental dysfunction. (ed. J.P. Aggleton), pp. 453-470. Wiley-Liss, NY.

Phelps, E.A., LaBar, K.S., and Spencer, D.D. 1997. Memory for emotional words following unilateral temporal lobectomy. Brain Cogn. 35: 85-109.

Pillon, B., Bazin, B., Deweer, B., Ehrle, N., Baulac, M., and Dubois, B. 1999. Specificity of memory deficits after right or left temporal lobectomy. Cortex 35: 561-571.

Saykin, A.J., Robinson, L.J., Stafiniak, P., Kester, D.B., Gur, R.C., O'Conner, M.J., and Sperling, M.R. 1992. Neuropsychological changes after anterior temporal lobectomy: Acute effects on memory, language, and music. In The neuropsychology of epilepsy. (ed. T.L. Bennett ), pp. 263-290. Plenum, NY.

Siegel, S. and Castellan, N.J. 1988. Nonparametric statistics for the behavioral sciences. McGraw-Hill, Boston, MA.

Squire, L. 1987. Memory and brain. Oxford University Press, NY.

Talairach, J. and Tournoux, P. 1988. Co-Planar stereotaxic atlas of the buman brain, 3-dimensional proportional system: An approach to cerebral imaging. Thieme Medical Publishers, NY.

Tranel, D. 1996. The Iowa-Benton school of neuropsychological assessment. In Neuropsychological assessment of neuropsychiatric disorders. (ed. I. Grant and K.M. Adams), pp. 81-101. Oxford University Press, NY.

Vanderploeg, R.D., Curtiss, G., Schinka, J.A., and Lanham Jr., R.A. 2001. Material-specific memory in traumatic brain injury: Differential effects during acquisition, recall, and retention. Neuropsychology 15: 174-184.

Zola-Morgan, S., Squire, L.R., and Amaral, D.G. 1986. Human amnesia and the medial temporal region: enduring memory impairment following a bilateral lesion limited to field CA1 of the hippocampus. J. Neurosci. 6: $2950-2967$.

Zola-Morgan, S., Squire, L.R., Alvarez-Royo, P., and Clower, R.P. 1991. Independence of memory functions and emotional behavior: Separate contributions of the hippocampal formation and the amygdala. Hippocampus 1: 207-220.

Received March 9, 2001; accepted in revised form September 12, 2001. 


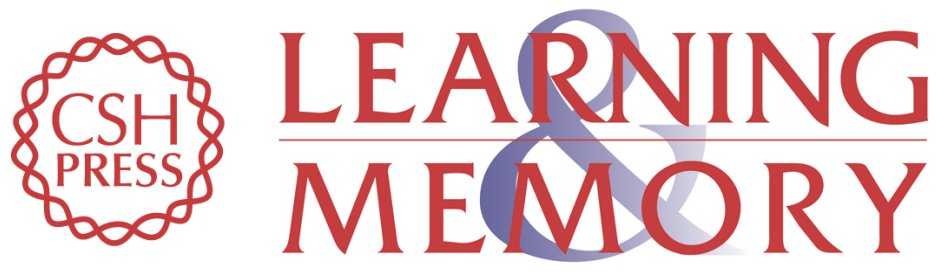

\section{Verbal and Nonverbal Emotional Memory Following Unilateral Amygdala Damage}

Tony W. Buchanan, Natalie L. Denburg, Daniel Tranel, et al.

Learn. Mem. 2001, 8:

Access the most recent version at doi:10.1101//m.40101

References This article cites 31 articles, 6 of which can be accessed free at: http://learnmem.cshlp.org/content/8/6/326.full.html\#ref-list-1

License

Email Alerting Receive free email alerts when new articles cite this article - sign up in the box at the Service top right corner of the article or click here. 